\title{
Evaluation of type 2 diabetes genetic risk variants in Chinese adults: findings from 93,000 individuals from the China Kadoorie Biobank
}

\author{
Wei Gan ${ }^{1,2} \cdot$ Robin G. Walters ${ }^{3} \cdot$ Michael V. Holmes ${ }^{3} \cdot$ Fiona Bragg $^{3} \cdot$

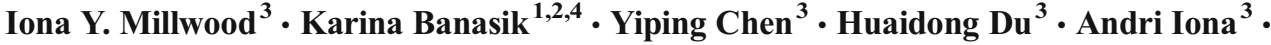 \\ Anubha Mahajan ${ }^{1,2} \cdot$ Ling Yang $^{3} \cdot$ Zheng Bian $^{5} \cdot$ Yu Guo $^{5} \cdot$ Robert J. Clarke $^{3}$. \\ Liming $\mathrm{Li}^{5,6}$ - Mark I. McCarthy ${ }^{1,2,7} \cdot$ Zhengming $\mathrm{Chen}^{3} \cdot$ on behalf of the China \\ Kadoorie Biobank Collaborative Group
}

Received: 29 December 2015 / Accepted: 22 February 2016 / Published online: 6 April 2016

(C) The Author(s) 2016. This article is published with open access at Springerlink.com

\begin{abstract}
Aims/hypothesis Genome-wide association studies (GWAS) have discovered many risk variants for type 2 diabetes. However, estimates of the contributions of risk variants to type 2 diabetes predisposition are often based on highly selected case-control samples, and reliable estimates of populationlevel effect sizes are missing, especially in non-European populations.

Methods The individual and cumulative effects of 59 established type 2 diabetes risk loci were measured in a population-based China Kadoorie Biobank (CKB) study of 93,000 Chinese adults, including >7,100 diabetes cases.

Results Association signals were directionally consistent between CKB and the original discovery GWAS: of 56 variants
\end{abstract}

Wei Gan and Robin G. Walters contributed equally to this study.

For a list of members of the China Kadoorie Biobank Collaborative Group, please see the ESM.

Electronic supplementary material The online version of this article (doi:10.1007/s00125-016-3920-9) contains peer-reviewed but unedited supplementary material, which is available to authorised users.

Mark I. McCarthy

mark.mccarthy@drl.ox.ac.uk

Zhengming Chen

zhengming.chen@ctsu.ox.ac.uk

1 Oxford Centre for Diabetes, Endocrinology, and Metabolism, University of Oxford, Churchill Hospital Campus, Old Road, Headington, Oxford OX3 7LJ, UK

2 Wellcome Trust Centre for Human Genetics, University of Oxford, Oxford, UK passing quality control, 48 showed the same direction of effect (binomial test, $p=2.3 \times 10^{-8}$ ). We observed a consistent overall trend towards lower risk variant effect sizes in CKB than in case-control samples of GWAS meta-analyses (mean 19-22\% decrease in log odds, $p \leq 0.0048$ ), likely to reflect correction of both 'winner's curse' and spectrum bias effects. The association with risk of diabetes of a genetic risk score, based on lead variants at 25 loci considered to act through beta cell function, demonstrated significant interactions with several measures of adiposity (BMI, waist circumference [WC], WHR and percentage body fat $[\mathrm{PBF}]$; all $p_{\text {interaction }}<1 \times 10^{-4}$ ), with a greater effect being observed in leaner adults.

Conclusions/interpretation Our study provides further evidence of shared genetic architecture for type 2 diabetes
3 Clinical Trial Service Unit and Epidemiological Studies Unit, University of Oxford, Old Road Campus, Oxford OX3 7LF, UK

4 The Novo Nordisk Foundation Center for Basic Metabolic Research, Section of Metabolic Genetics, University of Copenhagen, Copenhagen, Denmark

5 Chinese Academy of Medical Sciences, Dong Cheng District, Beijing, People's Republic of China

6 School of Public Health, Peking University Health Sciences Center, Beijing, People's Republic of China

7 National Institute of Health Research Oxford Biomedical Research Centre, Oxford, UK 
between Europeans and East Asians. It also indicates that even very large GWAS meta-analyses may be vulnerable to substantial inflation of effect size estimates, compared with those observed in large-scale population-based cohort studies.

Access to research materials Details of how to access China Kadoorie Biobank data and details of the data release schedule are available from www.ckbiobank.org/site/Data+Access.

Keywords Biobank · Chinese - Genetic risk score · Population-based cohort studies · Type 2 diabetes · Winner's curse

$\begin{array}{ll}\text { Abbreviations } & \\ \text { AGEN-T2D } & \begin{array}{l}\text { Asian Genetic Epidemiology Network- } \\ \text { Type 2 Diabetes Consortium }\end{array} \\ \text { CKB } & \text { China Kadoorie Biobank } \\ \text { GRS } & \text { Genetic risk score } \\ \text { GRS-BC } & \text { Beta cell function related genetic risk } \\ & \text { score } \\ \text { GRS-IR } & \text { Insulin resistance related genetic risk } \\ \text { GRS-T } & \text { score } \\ \text { GWAS } & \text { Overall genetic risk score } \\ \text { HOMA-B } & \text { Genome-wide association studies } \\ \text { IR } & \text { HOMA of beta cell function } \\ \text { LD } & \text { Insulin resistance } \\ \text { MAF } & \text { Linkage disequilibrium } \\ \text { PBF } & \text { Minor allele frequency } \\ \text { QC } & \text { Percentage body fat } \\ \text { ROC } & \text { Quality control } \\ \text { SNP } & \text { Receiver operating characteristic } \\ \text { TransEthnic } & \text { Single nucleotide polymorphism } \\ \text { TransEthnic }+ \text { CKB } & \text { Trans-ethnic type 2 diabetes GWAS } \\ \text { meta-analysis } & \text { Combined meta-analysis of the CKB } \\ \text { WC } & \text { and trans-ethnic GWAS studies } \\ & \text { Waist circumference } \\ & \end{array}$

\section{Introduction}

Type 2 diabetes affects $\sim 400$ million people globally [1]. The prevalence of type 2 diabetes has increased substantially in Asian populations, and in China it is estimated that 100 million adults ( $\sim 11 \%$ of the adult population) are affected [2]. Lifestyle factors (e.g. physical inactivity), nutrition transitions and increased adiposity are the chief determinants of type 2 diabetes, but genetic factors also play an important role.

Genome-wide association studies (GWAS) and large-scale genotyping studies (e.g. MetaboChip and ExomeChip genotyping arrays) have identified more than 90 type 2 diabetes associated risk loci [3-28]. GWAS and replication studies conducted in a range of ancestry groups have revealed that most common-variant susceptibility loci are shared across ethnic groups $[24,29,30]$. While many type 2 diabetes susceptibility variants identified in Europeans have been successfully replicated in East Asians, failure to replicate (e.g. at $A D C Y 5$, NOTCH 2 and $P R C 1$ ) likely arises from poor coverage by genotyping arrays, ethnic differences in allele frequency, variable linkage disequilibrium (LD) and limited statistical power.

As the number of type 2 diabetes associated variants has increased, so has the value in including genetic data in models to predict type 2 diabetes risk, weighting individual genetic variants according to their reported effect size [31]. However, effect estimates obtained from GWAS using case-control studies are often inflated due to spectrum bias and/or 'winner's curse' $[32,33]$. Spectrum bias describes the overestimation of test performance that can arise from studying 'clearcut' cases or extremes of the underlying distribution (so-called 'extreme phenotypes') [32]. 'Winner's curse' refers to the upward bias in the estimated effect of a newly identified variant, particularly when there is limited power to detect the true association [33]. To avoid these biases, large-scale population-based studies are required to obtain robust populationspecific estimates of both individual and joint effects of GWAS-identified variants. The availability of such data remains limited, especially in non-European populations, and this motivated us to obtain population-based estimates of effect size in the China Kadoorie Biobank (CKB) study.

In addition, we constructed genetic risk scores (GRSs) to investigate the separate genetic effects on diabetes of SNPs that have been associated with beta cell dysfunction or insulin resistance (IR). The association between certain genetic variants and type 2 diabetes risk has been reported to vary according to obesity status [34], which could impact on the utility of predictive models. Therefore, we assessed whether associations of these GRSs with diabetes were modified in individuals with different degrees of adiposity.

\section{Methods}

Study population The study sample consisted of 93,131 individuals with genotype data, randomly selected from the CKB study (www.ckbiobank.org), a prospective cohort of 512,891 Chinese adults. Details of the study design, protocol, procedures and characteristics of CKB have been described elsewhere [35]. Briefly, the baseline survey took place from June 2004 to July 2008 in ten geographically defined areas ( 5 urban, 5 rural) across China. In each study area, permanent residents were identified through official residential records, and invited to participate in the study. All participants are prospectively followed up for cause-specific mortality, morbidity and hospitalisation, using China CDC's Disease Surveillance Points and linkages to the national health insurance claim databases [35]. Information about socio- 
demographic, lifestyle, medical history and current medication were collected by laptop-based questionnaires. Physical measurements were recorded including height, weight, waist and hip circumferences, and bio-impedance (Tanita BC418MA, Tokyo, Japan). Except in one study area, where the protocol included fasting by all participants, initial screening for hyperglycaemia involved immediate on-site testing of non-fasting blood glucose using the SureStep Plus meter (LifeScan, Milpitas, CA, USA). Participants with nonfasting glucose levels $\geq 7.8$ and $<11.1 \mathrm{mmol} / 1$ were invited to return for a fasting blood glucose test the next day.

Diabetes was defined either as a self-report of physician diagnosis of diabetes or screen-detected diabetes, as previously reported [36]. For self-reported diabetes, those with an onset under age 30 and currently treated with insulin were considered as type 1 diabetes, and were excluded from the present analyses. Screen-detected diabetes was defined as no prior history of diabetes with a blood glucose level meeting any one of the following criteria, if applicable: (1) a random blood glucose level $\geq 7.0 \mathrm{mmol} / \mathrm{l}$ and a fasting time $>8 \mathrm{~h}$; (2) a random blood glucose level $\geq 11.1 \mathrm{mmol} / 1$ and a fasting time $<8 \mathrm{~h}$; (3) a fasting blood glucose level $\geq 7.0 \mathrm{mmol} / \mathrm{l}$. For the current analysis, follow-up data were collected up to 31 December 2013 (Snapshot Database Release 9, April 2015). We combined all cases of prevalent $(5,483)$ and incident $(1,626)$ diabetes to give a total of 7,109 cases and 86,022 non-diabetes controls. All participants provided written informed consent for follow-up and long-term storage of biological samples.

Genotyping A panel of 384 single nucleotide polymorphisms (SNPs), selected on the basis of prior association with cardiovascular disease, risk factors and related phenotypes, were genotyped in 95,680 randomly selected individuals from CKB on the Illumina Golden Gate platform at the BGI laboratory in Shenzhen, China. A total of 93,131 individuals aged 30-79 years passed quality control criteria (call rate $\geq 98 \%$, no sex mismatch, heterozygosity F statistic SD score $<5$ ). SNPs with low call rate $(<95 \%)$ or Hardy-Weinberg disequilibrium $\left(p<0.05 / 384=1.3 \times 10^{-4}\right)$ were excluded. Mean genotyping concordance was $99.98 \%$ (range $98.66-100 \%$ ) based on 2,063 duplicate samples included for quality control (QC) purposes. The SNP panel included 59 GWAS-identified type 2 diabetes risk variants reported by October 2012, of which five were originally reported in South Asians, 15 in East Asians and 36 in Europeans. These lead SNPs were selected based on the available association data from East Asian populations and/or fine-mapping data in Europeans at the time of array design and manufacturing. Since $H N F 1 A$ rs12427353 is monomorphic in East Asians and genotyping of two variants (PEPD rs3786897, KCNK16 rs3734618) failed QC, data were available for 56 variants (Fig. 1). The majority of SNPs were successfully genotyped in all selected samples except for four (WFS1 rs10010131, DGKB rs2191349, RASGRP1 rs7403531
59 GWAS-identified T2D SNPs by October 2012 (93,131 Chinese adults from CKB study)

3 SNPs excluded:

Monomorphic: HNF1A rs7957197

Genotype failure: PEPD rs3786897

KCNK16 rs3734618

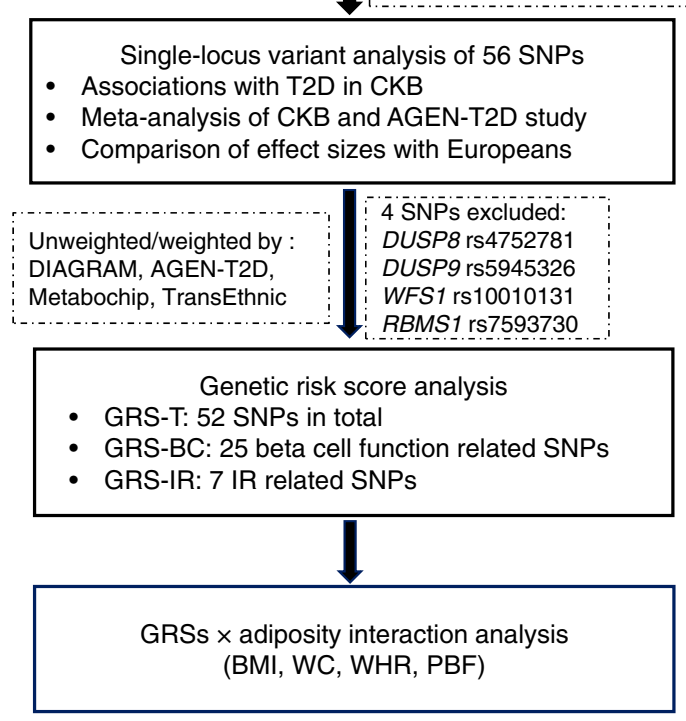

Fig. 1 Flow chart of the analyses. T2D, type 2 diabetes

and GRK5 rs10886471), which were genotyped only in batches comprising subsets of the cohort $(49 \%, 80 \%, 90 \%$ and $90 \%$ of participants, respectively). Estimates of relatedness based on 235 independent SNPs, using the R package SNPRelate [37], identified that $19 \%$ of participants had at least one first-degree relative among those genotyped. We present the results for the full dataset, but exclusion of 10,654 participants to eliminate first-degree relationships had no appreciable effect on individual results or our overall conclusions. Genomic inflation was estimated at $\lambda_{1000}=1.06-1.08$, based on associations for 235 independent SNPs.

GRSs The risk variants at DUSP8/INS, DUSP9 and WFS1 were not included in GRS calculations because of parent-oforigin-specific effects, location on the X-chromosome and low genotyping rate, respectively. RBMS1 rs 7593730 was also excluded as it was associated with type 2 diabetes only in Europeans. The remaining 52 variants were selected for the overall GRS (GRS-T) (Fig. 1 and Electronic Supplementary Material [ESM] Tables 1,2). Five types of weighted GRS (using weights derived from the natural logarithm of the perallele OR) were calculated, using data from: (1) DIAGRAMv3 GWAS meta-analysis (DIAGRAMv3) [15]; (2) GWAS metaanalysis in East Asians (Asian Genetic Epidemiology Network-Type 2 Diabetes Consortium [AGEN-T2D]) [14]; (3) DIAGRAM Metabochip meta-analysis (Metabochip) [15]; (4) a trans-ethnic type 2 diabetes GWAS meta-analysis (TransEthnic) [24]; and (5) a combined meta-analysis of the 
CKB and trans-ethnic GWAS studies (TransEthnic + CKB) (ESM Fig. 1).

Type 2 diabetes risk variants were classified, based on previously published data concerning their pathophysiological mechanism, as being predominantly related to beta cell dysfunction, IR or neither (ESM Table 1). We updated the strategy proposed by Vassy et al [38] by including more lines of genetic and physiological evidence [15, 39-41]. Beta cell dysfunction related SNPs were identified by: (1) association with decreased HOMA of beta cell function (HOMA-B; $p<0.05, \beta$ for HOMA-B $<0$ for risk allele) in non-diabetic individuals [15]; (2) association with one of the beta cell function indices during an OGTT ( $p<0.05, \beta<0$ for risk allele) [40, 41]; (3) presence in a locus influencing beta cell function according to cluster analysis [40]; and/or (4) the existence of rare variants responsible for forms of monogenic diabetes characterised by insulin secretory failure (such as neonatal diabetes and MODY). IR-related SNPs were identified by: (1) association with increased HOMA-IR $(p<0.05, \beta>0$ for risk allele) in non-diabetic individuals [41] or decreased insulin sensitivity index ( $p<0.05, \beta<0$ for risk allele) [15]; (2) association with fasting insulin $(p<0.05, \beta>0)$ [39]; (3) presence in a locus influencing insulin sensitivity according to cluster analysis [40]; (4) association with increased triacylglycerol or other IR-related traits [39]; and (5) not acting primarily through obesity (FTO rs9939609, MC4R rs12970134) [15]. Thus, GRSs were constructed from 25 beta cell dysfunction related SNPs (GRS-BC) and seven IR-related SNPs (GRS-IR) (ESM Table 2). Missing genotypes were imputed by assigning the mean genotype for that participant's regional centre. To make the weighted GRSs easier to interpret and more directly comparable to the unweighted score, values were rescaled as follows: $\mathrm{GRS}^{\prime}=\mathrm{GRS} \times$ total number of the risk alleles $/(2 \times$ sum of weights). Each point of the rescaled GRS thus corresponded to, on average, one additional risk allele.

Statistical analysis Departure from Hardy-Weinberg equilibrium was assessed using a $1-d f \chi^{2}$ test. For the primary outcome, logistic regression was used to estimate ORs and 95\% CIs of individual variants and GRSs for combined prevalent/ incident diabetes, adjusting for age, sex and regional centre. Comparison of effect sizes ( $\log _{\mathrm{e}} \mathrm{ORs}$ ) between CKB and previous studies was performed by inverse-variance weighted least squares regression through the origin. To combine our results with those from AGEN-T2D [14] or the TransEthnic meta-analysis [24], fixed effects meta-analysis was performed by inverse-variance weighting. We carefully checked the region of recruitment of the studies contributing to AGEN-T2D and found no evidence of overlap with CKB. Floating absolute risks were used to provide estimates of variance across GRS quartiles [42]. BMI cut-point categories were defined according to Asian criteria proposed by the WHO: normal weight $\left(\mathrm{BMI}<23 \mathrm{~kg} / \mathrm{m}^{2}\right)$; overweight $(23 \leq \mathrm{BMI}<27.5$ $\mathrm{kg} / \mathrm{m}^{2}$ ); obese $\left(\mathrm{BMI} \geq 27.5 \mathrm{~kg} / \mathrm{m}^{2}\right)$ [43]. Strata of waist circumference (WC), WHR and percentage body fat (PBF) were defined by sex-specific tertiles. Tests for interaction between adiposity and GRSs used logistic regression models including GRS, adiposity variable of interest and GRS $\times$ variable interaction term, with additional adjustment for age, sex and regional centre. Given that all SNPs were previously identified at GWAS significance for type 2 diabetes in Europeans and/or Asians, conventional Bonferroni correction would be overly conservative; we used the Holm-Bonferroni method or permutation procedures to control the family-wise error rate. For completeness, we also present findings using a $\%$ false discovery rate (Benjamini-Hochberg). In the meta-analyses, Cochran's Q test was used to assess between-study heterogeneity and Bonferroni correction was used to account for multiple testing $\left(p<0.05 /[55 \times 3]=3.0 \times 10^{-4}\right)$. The discriminative abilities of unweighted and weighted GRSs for risk of diabetes were assessed by receiver operating characteristic (ROC) curve analysis and compared using the DeLong test [44]. For 1000-fold cross-validation, weights were derived from a repeated analysis excluding randomly selected sets of $0.1 \%$ of the CKB sample, and using the resulting weights for the excluded individuals. The proportion of variance in phenotype explained for each SNP or GRS was calculated according to Shim et al [45] using previously reported means and SEs for SNP effect sizes [15]. We estimated power using ORs reported in the original GWAS and sample size and risk allele frequencies of our study with Quanto software (http://biostats.usc. edu/Quanto.html) (ESM Table 3). We investigated regional LD patterns among East Asians (CHB + JPT panel) and Europeans (CEU panel) from HapMap release 27 using the varLD algorithm [46], and presented results as Monte-Carlo $p$ values from 10,000 iterations. All reported $p$ values are nominal and 2-sided. Association analyses were performed using R software version 3.0.2 (www.r-project.org).

\section{Results}

Participant characteristics Among the 93,131 CKB participants, there were 7,109 (7.6\%) diabetes cases comprising 2,903 (3.1\%) self-reported and 2,580 (2.8\%) screen-detected at baseline, and 1,626 (1.7\%) incident cases of diabetes that occurred during a mean (SD) of 7.1 (1.3) years follow-up (Table 1). A total of 86,022 participants without diabetes were considered controls. The overall mean BMI was $23.6 \mathrm{~kg} / \mathrm{m}^{2}$. Women had slightly higher BMI than men, and also had higher prevalence and incidence of diabetes.

Association with individual variants in the CKB study Table 2 shows the associations of 56 variants that passed QC with diabetes risk, together with the corresponding values from AGEN-T2D and a meta-analysis of CKB and AGEN- 
Table 1 Selected characteristics at baseline among 93,131 genotyped participants in $\mathrm{CKB}$

\begin{tabular}{llll}
\hline Variable & Men & Women & All \\
\hline Individuals, $n(\%)$ & $37,677(40.5)$ & $55,454(59.5)$ & 93,131 \\
Age, years & $52.3(10.8)$ & $50.7(10.5)$ & $51.4(10.7)$ \\
Random blood glucose $^{\mathrm{a}}, \mathrm{mmol} / \mathrm{l}$ & $6.0(2.4)$ & $6.2(2.4)$ & $6.1(2.4)$ \\
WC, cm & $82.0(9.7)$ & $79.1(9.6)$ & $80.3(9.8)$ \\
Hip circumference, cm & $90.6(6.8)$ & $91.1(6.9)$ & $90.9(6.9)$ \\
BMI, kg/m & $23.4(3.2)$ & $23.8(3.5)$ & $23.6(3.4)$ \\
WHR & $0.90(0.06)$ & $0.87(0.07)$ & $0.88(0.07)$ \\
PBF, \% & $21.9(6.2)$ & $32.1(7.2)$ & $28.1(8.4)$ \\
Diabetes, $n(\%)$ & $2,678(7.1)$ & $4,431(8.0)$ & $7,109(7.6)$ \\
$\quad$ Clinically identified & $1,101(2.9)$ & $1,802(3.3)$ & $2,903(3.1)$ \\
$\quad$ Screen-detected & $991(2.6)$ & $1,589(2.9)$ & $2,580(2.8)$ \\
$\quad$ Incident & $586(1.6)$ & $1,040(1.9)$ & $1,626(1.8)$ \\
\hline
\end{tabular}

Data are means (SD) or $n(\%)$

${ }^{a}$ Data only available for 92,166 participants (Men: 37,257; Women: 54,909)
T2D. Risk allele frequencies observed in $\mathrm{CKB}$ were comparable with those in the 1000 Genomes Project Phase $3 \mathrm{CHB}+$ CHS population. Sensitivity analysis showed that there was no evidence of heterogeneity between regional centres in the association of loci and risk of diabetes (ESM Tables 4-6). All SNPs were common in CKB (minor allele frequency, MAF $>0.05$ ) except for variants at five loci: (NOTCH2 rs10923931 [MAF =0.032]; THADA rs7578597 [0.007]; ADCY5 rs11708067 [0.003]; TCF7L2 rs7901695 [0.031]; and PRC1 rs8042680 [0.010]). Among the 56 variants, 48 had effects directionally consistent with those in the original reports (binomial test, $\left.p=2.3 \times 10^{-8}\right)(\mathrm{ESM}$ Tables 7,8$)$. Five SNPs reached GWAS significance $\left(p<5 \times 10^{-8}\right)$ and another 14 variants showed statistically significant association after multiple-testing correction (Holm-Bonferroni, $p<0.05$ ); alternatively, association was replicated for 30 SNPs at $5 \%$ false discovery rate (Benjamini-Hochberg). All eight risk loci that were identified in East Asian and Chinese Han GWAS $[14,18]$ showed consistent effect directions. Among them, $M A E A$ and GLIS3 loci were significantly associated with type 2 diabetes after correction for multiple testing.

Meta-analysis of CKB and AGEN-T2D studies Metaanalysis combining the results of the present study with those from AGEN-T2D [14], providing a total of 32,188 cases and 115,633 controls, further improved concordance of effect estimates: after excluding variants identified in AGEN-T2D or its contributing cohorts, 37 of 40 variants were directionally consistent with European populations (binomial test $p=9.73 \times 10^{-9}$ ). Ten variants identified in GWAS studies of Europeans, plus nine variants reported in East Asian GWAS studies, were genome-wide significant (Table 2, ESM Tables 7,8). With the exception of ZFAND3, we found no heterogeneity for the associations at these loci across $\mathrm{CKB}$ and AGEN-T2D (Table 2).
Consistency of effect sizes between East Asians and Europeans Allelic ORs estimated in CKB were highly correlated with those from Europeans (Fig. 2, $r=0.81, p=2.1 \times 10^{-36}$ ). However, there was a clear trend towards lower effect sizes in this population-based study of Chinese than in the predominantly case-control samples of European descent included in GWAS discovery studies, with a mean proportional reduction in $\log _{\mathrm{e}} \mathrm{OR}$ of $19 \%\left(95 \%\right.$ CI 6,$\left.32 ; p=4.8 \times 10^{-3}\right)$. A very similar reduction in effect size was observed when comparing CKB and AGENT2D, which also comprised predominantly case-control cohorts ( $22 \% ; p=3.4 \times 10^{-3}$, ESM Fig. 2a). Effect sizes were also strongly correlated when comparing the meta-analysis of $\mathrm{CKB}$ and AGEN-T2D with Europeans $\left(r=0.85, p=7.6 \times 10^{-37}\right.$; proportional reduction of $15 \%, p=0.026$ ) (ESM Fig. 2b).

Heterogeneity in effect size was observed at RBMS1 rs7593730 and GCC1-PAX4 rs6467136 $\left(p<10^{-4}\right)$ (ESM Table 9), whose associations with diabetes were significant only in Europeans and East Asians, respectively. This potentially reflects the different LD patterns between East Asians and Europeans at these loci $(p<0.0002$ for both, ESM Table 10). A further large difference in estimated effect size between CKB (or AGEN-T2D + CKB) and Europeans, for ADCY5 rs11708067 (OR [95\% CI]: 1.92 [1.28, 2.88] vs 1.10 $[1.06,1.15])$, likely reflects low power and uncertainty in effect size in CKB: neither the difference nor the diabetes association itself was significant after correction for multiple testing. This SNP shows large differences in MAF (0.003 and 0.217 in Chinese and Europeans, respectively). In general, however, risk allele frequencies were similar in CKB and Europeans (CEU) (ESM Fig. 3, $r=0.62, p=2.9 \times 10^{-7}$ ).

GRSs and type 2 diabetes risk prediction $\mathrm{ROC}$ analysis to assess prediction of diabetes in CKB by GRS-T based on 52 type 2 diabetes risk variants genotyped in the majority of samples showed that, compared with the unweighted risk score 


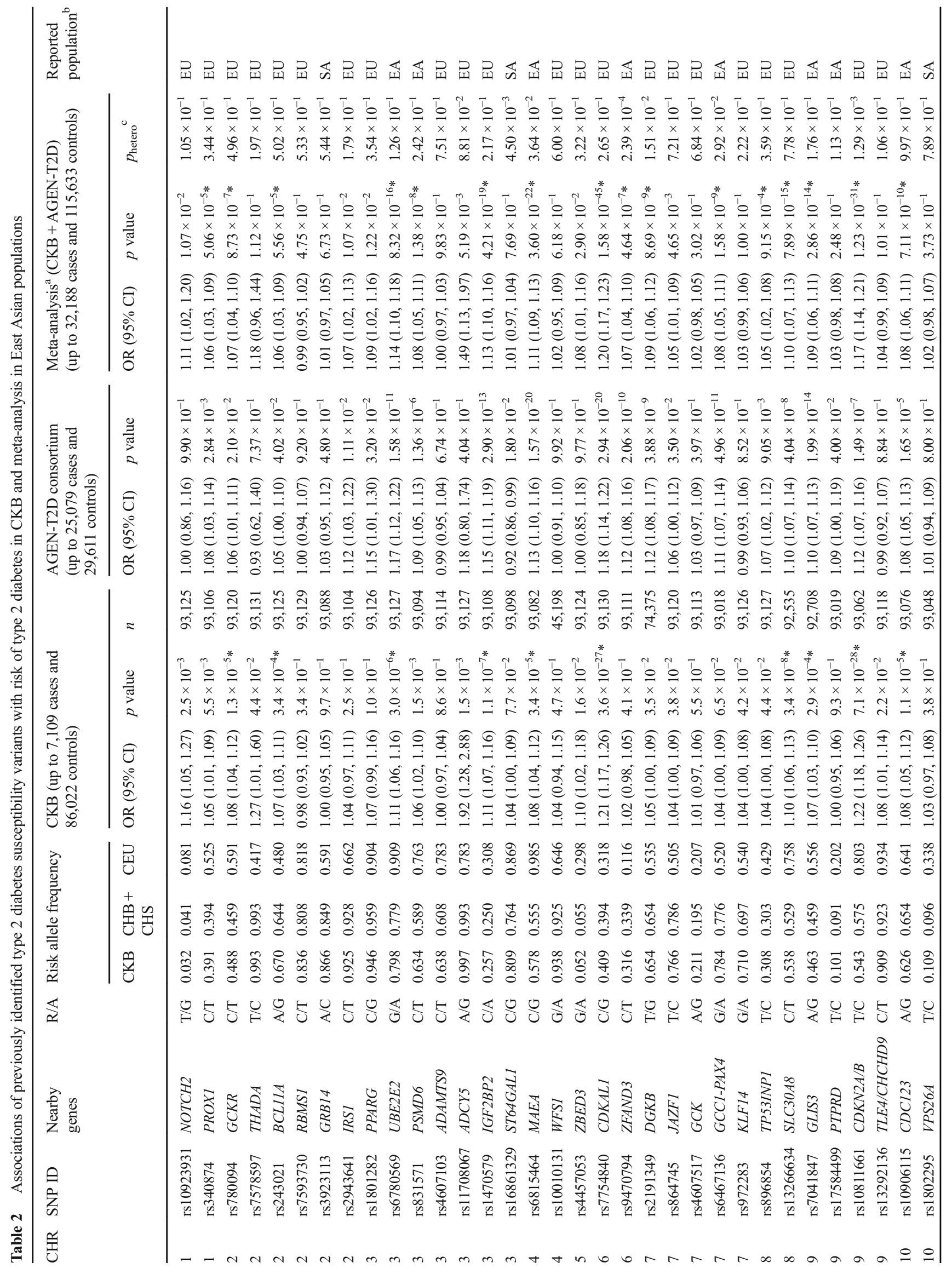




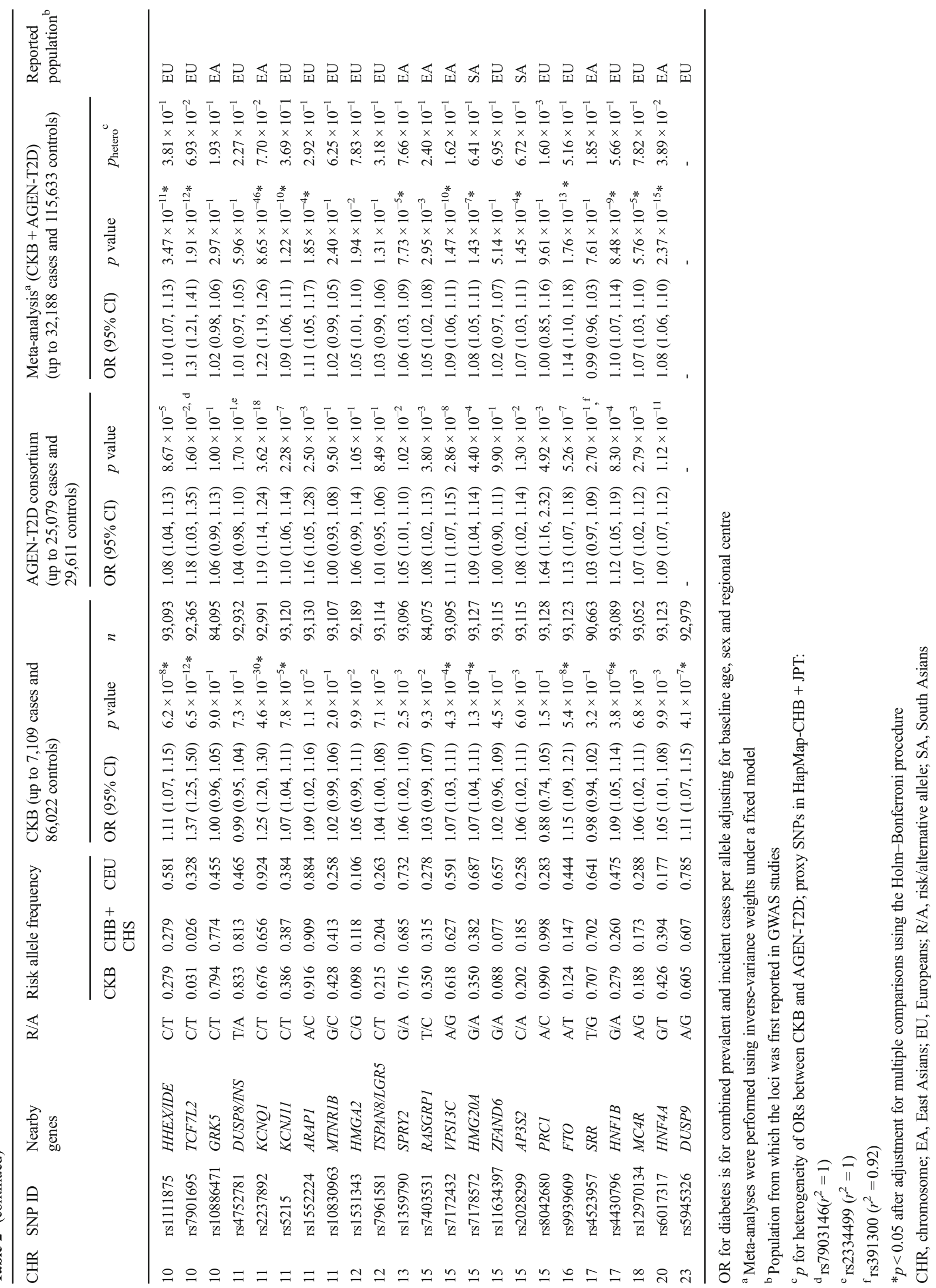


Fig. 2 Comparison estimates of effect size $\left(\log _{\mathrm{e}}(\mathrm{OR})\right)$ between Chinese and Europeans. The solid line represents the line of equality, and the result of regression is shown as a broken line with $95 \%$ CI

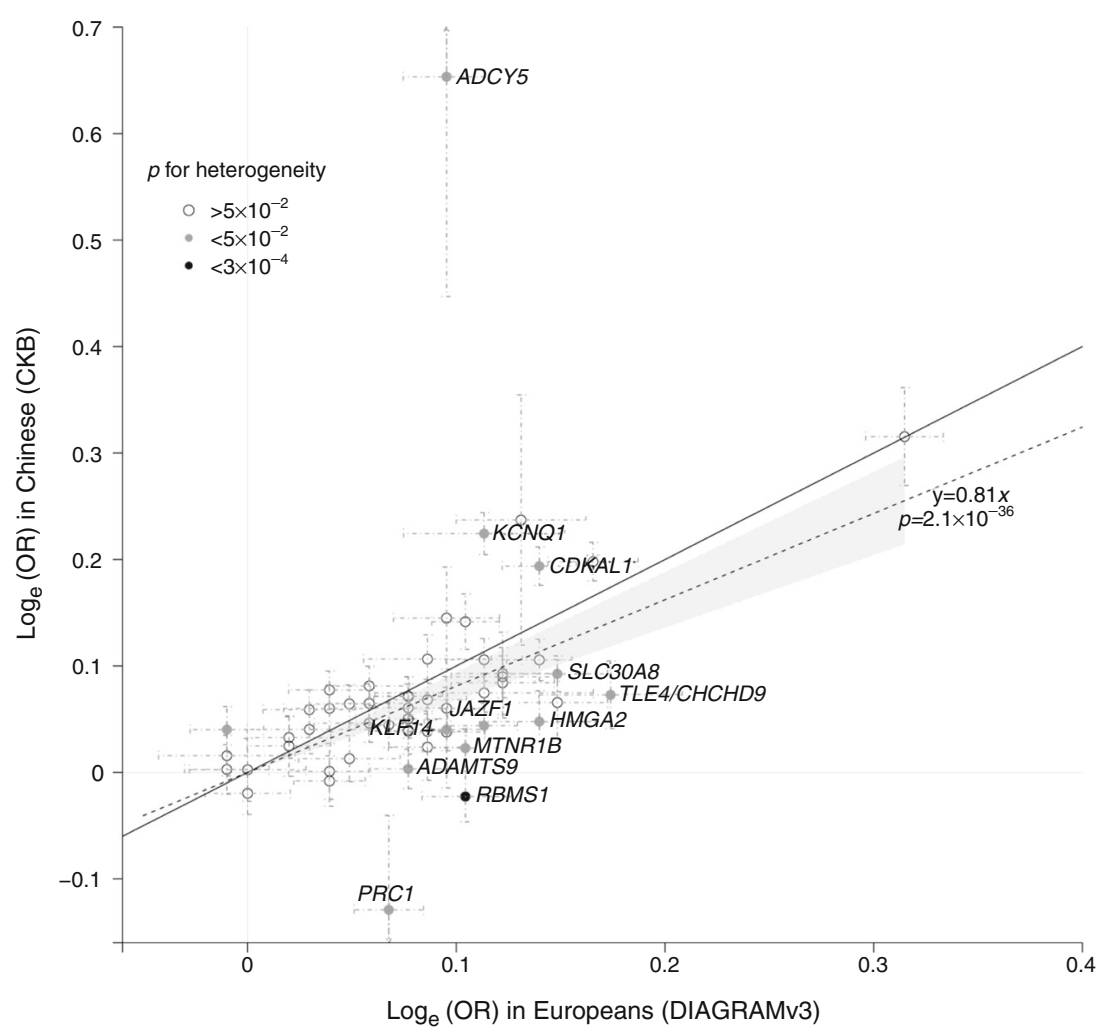

(C statistic [95\% CI]: 0.574 [0.567, 0.580]), there were significant improvements in discrimination when using risk scores based on weights from previous meta-analyses, TransEthnic in particular $\left(0.590\right.$ [0.583, 0.597], $p=3.6 \times 10^{-20}$, TransEthnic vs unweighted). There was a further small but significant improvement in diabetes prediction by GRS-T using weights from a meta-analysis including CKB (TransEthnic + CKB) $(0.593$ $[0.586,0.600] ; p=3.0 \times 10^{-12}$, TransEthnic + CKB vs TransEthnic) (Fig. 3 and ESM Table 11). Although somewhat reduced, there remained an improvement following 1000-fold cross-validation to minimise 'over-fitting' (0.591 [0.584, $\left.0.598], p=1.8 \times 10^{-3}\right)$. Thus, in terms of diabetes prediction/ discrimination this TransEthnic + CKB meta-analysis (ESM Tables 2,11$)$ provides the best-performing currently available estimates of effect size for these type 2 diabetes associated SNPs.

As expected, both unweighted and weighted GRS robustly associated with risk of diabetes (ESM Table 12). Individuals in the highest quartile of GRS-T had an OR of $2.34(2.25,2.45)$ compared with the lowest quartile. Note that, for this and subsequent analyses, we present the results of analyses employing TransEthnic-weighted GRSs, which represent the best-performing external weights - i.e. which avoid potential over-fitting and, therefore, do not require unnecessarily complex cross-validation analyses. Conclusions were not materially affected by using unweighted risk scores or other externally weighted scores (ESM Table 12).
To investigate the genetic contribution to diabetes related to beta cell dysfunction or IR, two separate GRSs (GRS-BC and GRS-IR) were calculated based on 25 variants predominantly associated with beta cell dysfunction and seven variants with IR, respectively. Assessments of the proportion of variances in HOMA-B and HOMA-IR explained by these GRSs confirmed

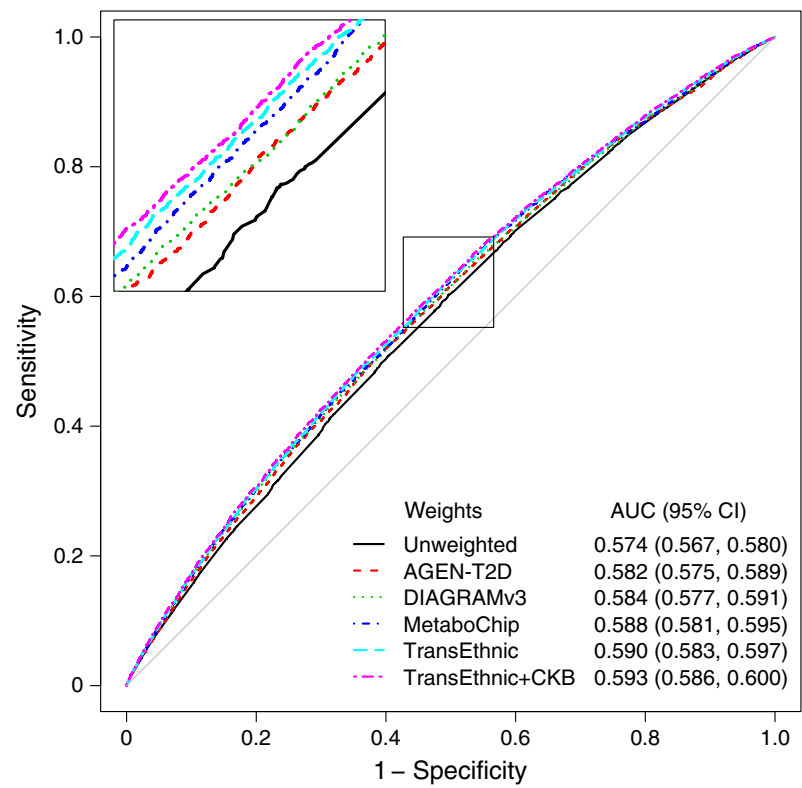

Fig. 3 ROC curves for type 2 diabetes GRS-Ts predicting type 2 diabetes in $\mathrm{CKB}$ 
that they successfully targeted the phenotype of interest (ESM Table 13). We identified associations of both GRS-BC and GRS-IR with diabetes (ESM Table 12). The ORs for diabetes were $2.17(2.08,2.26)$ and $1.19(1.14,1.25)$ when comparing the extreme quartiles of TransEthnic-weighted GRS-BC and GRSIR, respectively ( $p$ for trend $=4.82 \times 10^{-111}$ and $1.68 \times 10^{-7}$ ) (ESM Fig. 4).

Effect modification by adiposity Stratified analyses were performed to investigate the possible modifying effects of adiposity on the associations of GRSs with diabetes. Both GRS-T and GRS-BC showed significant interactions with strata for each of BMI, WC, WHR and PBF $\left(p_{\text {interaction }}<1 \times 10^{-4}\right)($ Table 3, ESM Table 15). In each case, per unit GRS score effects were greater in participants who were leaner. We also observed a strong inverse association of GRS-BC with BMI, WC and PBF, but not with WHR (ESM Table 15). In contrast, we found no evidence of interaction between GRS-IR and any of BMI, WC, WHR or PBF $\left(p_{\text {interaction }} \geq 0.11\right)$.

\section{Discussion}

We tested associations of 56 variants with risk of diabetes in a large-scale population-based study of Chinese adults. The effect sizes for the majority of diabetes loci were broadly similar between Chinese and European populations. However, there was an overall tendency towards lower effect sizes in our unselected population, likely the consequence of reduced bias (spectrum bias and 'winner's curse'). Similar reductions in effect size were observed when comparing with previous data from East Asians, so this is unlikely to be a reflection of differences in patterns of LD.

Improved estimates of SNP effect sizes enabled construction of more accurate weighted GRS for disease prediction. Although GRSs alone remain relatively poor predictors of diabetes risk compared with traditional risk models, the increasing numbers of associated SNPs nevertheless afford improvements for risk prediction [31]. Optimally, integration of genotyping data into type 2 diabetes risk prediction models requires reliable, unbiased, population-specific estimates of the effect of risk variants. Most current effect size estimates have been derived from gene discovery studies largely involving case-control samples and may suffer from 'winner's curse' and disease spectrum bias, the latter not being overcome by ever-larger non-population-based cohort studies. Re-estimation in population-based cohort studies of the effects of GWAS-identified loci limits such biases [32, 33]. Thus, the results from this study can be extrapolated to the Chinese general population and used for inclusion of genetic data in type 2 diabetes risk prediction models.
Previous studies have reported that the majority of common variants are shared across different ethnic groups [24, 30, $47,48]$. We have provided further evidence for shared genetic architecture of type 2 diabetes between East Asian and European populations. Further meta-analysis of CKB with published data from AGEN-T2D improved our statistical power to replicate associations originally reported in other ancestries: the number of variants that achieved genomewide significance was increased from four to ten, and eight additional SNPs showed significant association after multipletesting correction (ESM Tables 7, 8).

There are several potential reasons for failure to replicate variants or inconsistencies of effect sizes. First, differences in allele frequency between the original discovery population and the replicating studies in other ethnic groups may affect power for replication. Second, as effect sizes of more-recently identified type 2 diabetes loci become smaller (facilitated by everlarger sample sizes for discovery), a correspondingly larger sample size is needed for replication. Thus, 19 out of the 26 SNPs with the largest effect sizes $(\mathrm{OR} \geq 1.08)$ but none of the remainder reached genome-wide significance in the CKBAGEN-T2D meta-analysis. Third, differing patterns of LD may mean that genotyped SNPs are less effective proxies for the underlying causal variant in East Asians than in Europeans: at most loci, the SNPs investigated in the present study were identified in European studies. However, this is likely to apply to only a small subset of loci since common-variant associations map to common haplotypes that are broadly shared between Europeans and East Asians [24, 30, 48]. Thus, at loci where there was apparent non-concordance of effects (e.g. RBMS1 rs7593730 and GCC1-PAX4 rs6467136), it remains possible that the same causal variant is present in the two populations and has similar effects. Fourth, these may represent rare instances of ethnic differences in the occurrence of particular causal variants. This may be the case in Europeans for GCC1-PAX4 rs6467136, for which locus there are no SNPs with a type 2 diabetes association at even $p<10^{-3}$ within $500 \mathrm{~kb}$ [15]. This may be elucidated by future fine-mapping and sequencing studies [30].

We further investigated the genetic influence on disease risk by stratifying on genetic variants related primarily to either beta cell function or insulin sensitivity. While variants identified in early GWAS are mainly implicated in beta cell function, more recent studies have identified several variants with a primary impact on IR [15, 40, 41]. Stratification of type 2 diabetes cases according to the separate contributions of genetic effects on beta cell function and IR has the potential to be informative for so-called 'precision medicine'.

Consistent with previous reports [10, 34, 49], we identified interactions of GRS with measures of adiposity: GRS-BC had a larger effect size on diabetes among individuals with lower BMI, WC, WHR or PBF. Since we observed a strong inverse association of GRS-BC with BMI, WC and PBF (ESM 


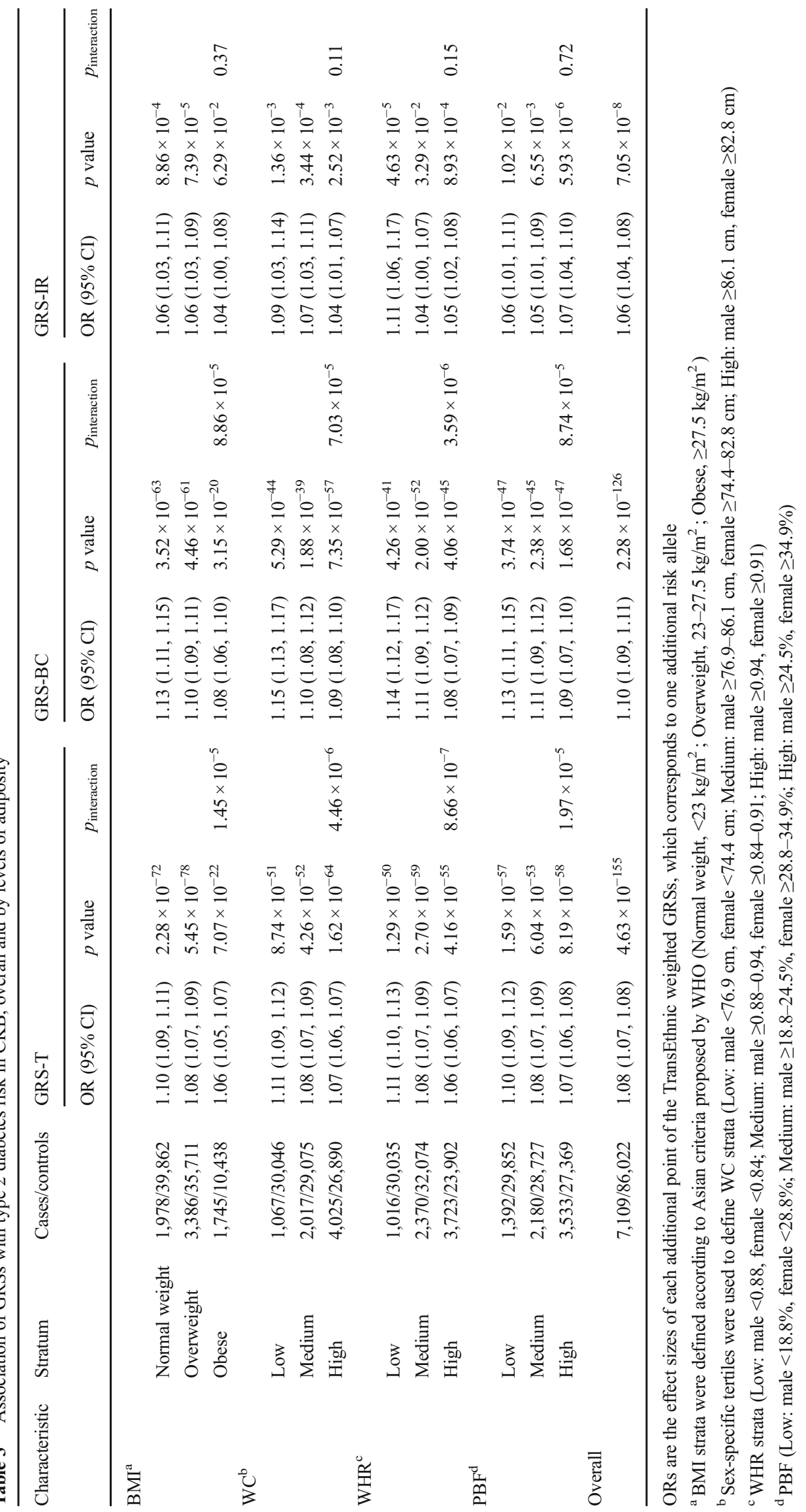


Table 15), it remains possible that the observed interactions with these measures of adiposity are an artefact of the dual effects of GRS-BC on both diabetes risk and adiposity, for instance due to 'collider bias' whereby analyses stratified by a potential mediator can induce new relationships and introduce confounding. However, this is not relevant for the interaction with WHR, which displayed a similar magnitude of interaction despite not showing association with GRS-BC. Therefore, we conclude that these observed effects of adiposity of GRS-BC effect size are likely to be genuine. By contrast, we found no evidence for interactions between GRS-IR and adiposity measures, which may reflect limited power (owing to GRS-IR comprising one-third the number of SNPs compared with GRS-BC and being less strongly associated with diabetes), but our findings are in line with previous findings that IR scores are associated with incident type 2 diabetes independent of body size [50].

In conclusion, we report estimates, expected to be largely free of 'winner's curse' and spectrum bias, of the effect sizes of diabetes risk variants in a general population cohort of Chinese adults. We thereby identify the extent to which previous GWAS based on predominantly case-control studies are affected by these biases. In addition to their utility for improvements in type 2 diabetes risk prediction, these more accurate effect size estimates promise to be a powerful resource for future Mendelian randomisation studies in Chinese cohorts.

Acknowledgements The chief acknowledgement is to the participants, the project staff, and the China National Centre for Disease Control and Prevention (CDC) and its regional offices for assisting with the fieldwork. We thank J. Mackay in Hong Kong; Y. Wang, G. Yang, Z. Qiang, L. Feng, M. Zhou, W. Zhao and Y. Zhang in China CDC; L. Kong, X. Yu and $\mathrm{K}$. Li in the Chinese Ministry of Health; and S. Clark, M. Radley, M. Hill, H. Pan and J. Boreham in the CTSU, Oxford, for assisting with the design, planning, organisation and conduct of the study.

Funding The CKB baseline survey and the first re-survey were supported by the Kadoorie Charitable Foundation in Hong Kong. The longterm follow-up is supported by the UK Wellcome Trust $(088158 / \mathrm{Z} / 09 / \mathrm{Z}$, 104085/Z/14/Z), Chinese Ministry of Science and Technology (2011BAI09B01), Chinese National Natural Science Foundation (81390541). The British Heart Foundation, UK Medical Research Council and Cancer Research UK provide core funding to the Clinical Trial Service Unit and Epidemiological Studies Unit at Oxford University for the project. Support for this analysis is provided by National Institute of Diabetes and Digestive and Kidney Diseases grant U01-DK085545. MM is a Wellcome Trust Senior Investigator, and is supported by Wellcome trust grant 098381. FB acknowledges support from the BHF Centre of Research Excellence, Oxford.

Contribution statement The study was conceived and designed by WG, RW, IM, RC, MM and ZC. WG performed data analysis and drafted the manuscript. RW and $\mathrm{MH}$ assisted with statistical analysis and contributed to the interpretation of the results. RW carried out SNP QC, clustering and genotyping calling. WG, FB, IM, HD, YC, LY, ZB and YG contributed to data acquisition and prepared the final phenotype database. $\mathrm{RW}, \mathrm{MH}, \mathrm{KB}, \mathrm{AI}$ and $\mathrm{AM}$ contributed to data analysis. LL and $\mathrm{ZC}$ designed the CKB study. All authors commented on and revised the manuscript. All authors gave final approval to the published version of this paper. ZC and RW are the guarantors of this work and, as such, had full access to all the data in the study and take responsibility for the integrity of the data and the accuracy of the data analysis.

Duality of interest The authors declare that there is no duality of interest associated with this manuscript.

Open Access This article is distributed under the terms of the Creative Commons Attribution 4.0 International License (http:// creativecommons.org/licenses/by/4.0/), which permits unrestricted use, distribution, and reproduction in any medium, provided you give appropriate credit to the original author(s) and the source, provide a link to the Creative Commons license, and indicate if changes were made.

\section{References}

1. WHO (2014) World Health Organization's annual World Health Statistics reports 2014. Available from www.who.int/gho/ publications/world health_statistics/, accessed 27 Feb 2015

2. Xu Y, Wang L, He J et al (2013) Prevalence and control of diabetes in Chinese adults. JAMA 310:948-959

3. Scott LJ, Mohlke KL, Bonnycastle LL et al (2007) A genome-wide association study of type 2 diabetes in Finns detects multiple susceptibility variants. Science $316: 1341-1345$

4. Zeggini E, Scott LJ, Saxena R et al (2008) Meta-analysis of genome-wide association data and large-scale replication identifies additional susceptibility loci for type 2 diabetes. Nat Genet 40:638645

5. Kong A, Steinthorsdottir V, Masson G et al (2009) Parental origin of sequence variants associated with complex diseases. Nature 462 : 868-874

6. Dupuis J, Langenberg C, Prokopenko I et al (2010) New genetic loci implicated in fasting glucose homeostasis and their impact on type 2 diabetes risk. Nat Genet 42:105-116

7. Qi L, Cornelis MC, Kraft P et al (2010) Genetic variants at 2q24 are associated with susceptibility to type 2 diabetes. Hum Mol Genet 19:2706-2715

8. Shu XO, Long J, Cai Q et al (2010) Identification of new genetic risk variants for type 2 diabetes. PLoS Genet 6:e1001127

9. Tsai FJ, Yang CF, Chen CC et al (2010) A genome-wide association study identifies susceptibility variants for type 2 diabetes in Han Chinese. PLoS Genet 6:e1000847

10. Voight BF, Scott LJ, Steinthorsdottir V et al (2010) Twelve type 2 diabetes susceptibility loci identified through large-scale association analysis. Nat Genet 42:579-589

11. Yamauchi T, Hara K, Maeda S et al (2010) A genome-wide association study in the Japanese population identifies susceptibility loci for type 2 diabetes at UBE2E2 and C2CD4A-C2CD4B. Nat Genet 42:864-868

12. Kooner JS, Saleheen D, Sim X et al (2011) Genome-wide association study in individuals of South Asian ancestry identifies six new type 2 diabetes susceptibility loci. Nat Genet 43:984-989

13. Parra EJ, Below JE, Krithika S et al (2011) Genome-wide association study of type 2 diabetes in a sample from Mexico City and a meta-analysis of a Mexican-American sample from Starr County, Texas. Diabetologia 54:2038-2046

14. Cho YS, Chen $\mathrm{CH}, \mathrm{Hu} \mathrm{C}$ et al (2012) Meta-analysis of genomewide association studies identifies eight new loci for type 2 diabetes in east Asians. Nat Genet 44:67-72 
15. Morris AP, Voight BF, Teslovich TM et al (2012) Large-scale association analysis provides insights into the genetic architecture and pathophysiology of type 2 diabetes. Nat Genet 44:981-990

16. Palmer ND, McDonough CW, Hicks PJ et al (2012) A genomewide association search for type 2 diabetes genes in African Americans. PLoS One 7:e29202

17. Saxena R, Elbers CC, Guo Y et al (2012) Large-scale gene-centric meta-analysis across 39 studies identifies type 2 diabetes loci. Am J Hum Genet 90:410-425

18. Li H, Gan W, Lu L et al (2013) A genome-wide association study identifies GRK5 and RASGRP1 as type 2 diabetes loci in Chinese Hans. Diabetes 62:291-298

19. Ma RC, Hu C, Tam $\mathrm{CH}$ et al (2013) Genome-wide association study in a Chinese population identifies a susceptibility locus for type 2 diabetes at 7q32 near PAX4. Diabetologia 56:1291-1305

20. Tabassum R, Chauhan G, Dwivedi OP et al (2013) Genome-wide association study for type 2 diabetes in Indians identifies a new susceptibility locus at 2q21. Diabetes 62:977-986

21. Consortium STD, Estrada K, Aukrust I et al (2014) Association of a low-frequency variant in HNF1A with type 2 diabetes in a Latino population. JAMA 311:2305-2314

22. Moltke I, Grarup N, Jorgensen ME et al (2014) A common Greenlandic TBC1D4 variant confers muscle insulin resistance and type 2 diabetes. Nature 512:190-193

23. Ng MC, Shriner D, Chen BH et al (2014) Meta-analysis of genomewide association studies in African Americans provides insights into the genetic architecture of type 2 diabetes. PLoS Genet 10: e1004517

24. Replication DIG, Meta-analysis C, Asian Genetic Epidemiology Network Type 2 Diabetes C et al (2014) Genome-wide trans-ancestry meta-analysis provides insight into the genetic architecture of type 2 diabetes susceptibility. Nat Genet 46:234-244

25. Wessel J, Chu AY, Willems SM et al (2015) Low-frequency and rare exome chip variants associate with fasting glucose and type 2 diabetes susceptibility. Nat Commun 6:5897

26. Consortium STD, Williams AL, Jacobs SB et al (2014) Sequence variants in SLC16A11 are a common risk factor for type 2 diabetes in Mexico. Nature 506:97-101

27. Mahajan A, Sim X, Ng HJ et al (2015) Identification and functional characterization of G6PC2 coding variants influencing glycemic traits define an effector transcript at the G6PC2-ABCB11 locus. PLoS Genet 11:e1004876

28. Imamura M, Maeda S, Yamauchi T et al (2012) A single-nucleotide polymorphism in ANK1 is associated with susceptibility to type 2 diabetes in Japanese populations. Hum Mol Genet 21:3042-3049

29. Carlson CS, Matise TC, North KE et al (2013) Generalization and dilution of association results from European GWAS in populations of non-European ancestry: the PAGE study. PLoS Biol 11: e1001661

30. Kuo JZ, Sheu WH, Assimes TL et al (2013) Trans-ethnic fine mapping identifies a novel independent locus at the 3 ' end of CDKAL1 and novel variants of several susceptibility loci for type 2 diabetes in a Han Chinese population. Diabetologia 56:26192628

31. Talmud PJ, Cooper JA, Morris RW et al (2015) Sixty-five common genetic variants and prediction of type 2 diabetes. Diabetes 64 : $1830-1840$

32. Ransohoff DF, Feinstein AR (1978) Problems of spectrum and bias in evaluating the efficacy of diagnostic tests. N Engl J Med 299: 926-930
33. Zollner S, Pritchard JK (2007) Overcoming the winner's curse: estimating penetrance parameters from case-control data. Am J Hum Genet 80:605-615

34. Perry JR, Voight BF, Yengo L et al (2012) Stratifying type 2 diabetes cases by BMI identifies genetic risk variants in LAMA1 and enrichment for risk variants in lean compared to obese cases. PLoS Genet 8:e1002741

35. Chen Z, Chen J, Collins R et al (2011) China Kadoorie Biobank of 0.5 million people: survey methods, baseline characteristics and long-term follow-up. Int J Epidemiol 40:1652-1666

36. Bragg F, Li L, Smith M et al (2014) Associations of blood glucose and prevalent diabetes with risk of cardiovascular disease in 500 000 adult Chinese: the China Kadoorie Biobank. Diabet Med 31: $540-551$

37. Zheng X, Levine D, Shen J, Gogarten SM, Laurie C, Weir BS (2012) A high-performance computing toolset for relatedness and principal component analysis of SNP data. Bioinformatics 28: 3326-3328

38. Vassy JL, Hivert MF, Porneala B et al (2014) Polygenic type 2 diabetes prediction at the limit of common variant detection. Diabetes 63:2172-2182

39. Manning AK, Hivert MF, Scott RA et al (2012) A genome-wide approach accounting for body mass index identifies genetic variants influencing fasting glycemic traits and insulin resistance. Nat Genet 44:659-669

40. Dimas AS, Lagou V, Barker A et al (2014) Impact of type 2 diabetes susceptibility variants on quantitative glycemic traits reveals mechanistic heterogeneity. Diabetes 63:2158-2171

41. Prokopenko I, Poon W, Magi R et al (2014) A central role for GRB10 in regulation of islet function in man. PLoS Genet 10: e1004235

42. Easton DF, Peto J, Babiker AG (1991) Floating absolute risk: an alternative to relative risk in survival and case-control analysis avoiding an arbitrary reference group. Stat Med 10:1025-1035

43. Consultation WHOE (2004) Appropriate body-mass index for Asian populations and its implications for policy and intervention strategies. Lancet 363:157-163

44. Robin X, Turck N, Hainard A et al (2011) pROC: an open-source package for $\mathrm{R}$ and $\mathrm{S}+$ to analyze and compare ROC curves. BMC Bioinforma 12:77

45. Shim H, Chasman DI, Smith JD et al (2015) A multivariate genome-wide association analysis of 10 LDL subfractions, and their response to statin treatment, in 1868 Caucasians. PLoS One 10:e0120758

46. Ong RT, Teo YY (2010) varLD: a program for quantifying variation in linkage disequilibrium patterns between populations. Bioinformatics 26:1269-1270

47. Sim X, Ong RT, Suo C et al (2011) Transferability of type 2 diabetes implicated loci in multi-ethnic cohorts from Southeast Asia. PLoS Genet 7:e1001363

48. Marigorta UM, Navarro A (2013) High trans-ethnic replicability of GWAS results implies common causal variants. PLoS Genet 9, e1003566

49. Langenberg C, Sharp SJ, Franks PW et al (2014) Gene-lifestyle interaction and type 2 diabetes: the EPIC interact case-cohort study. PLoS Med 11:e1001647

50. Scott RA, Fall T, Pasko D et al (2014) Common genetic variants highlight the role of insulin resistance and body fat distribution in type 2 diabetes, independent of obesity. Diabetes 63:4378-4387 